\title{
Effect of tamsulosin on ejaculatory function in BPH/LUTS
}

\author{
Sang Hoon Song ${ }^{1}$, Hwancheol Son ${ }^{2}$, Kwang Taek Kim ${ }^{3}$, Sae Woong Kim ${ }^{4}$, Du Geon Moon ${ }^{5}$, Ki Hak Moon ${ }^{6}$, \\ Kwangsung Park ${ }^{7}$, Jong Kwan Park ${ }^{8,9}$, Sung-Won Lee ${ }^{10}$, Jae Seog Hyun ${ }^{11}$ and Nam Cheol Park ${ }^{12}$
}

This study was undertaken to determine the impact on ejaculatory function of tamsulosin (0.2 mg) given once daily (OD) for 12 weeks and to identify risk factors for ejaculatory dysfunction in patients undergoing this treatment. Males with an International Prostatic Symptom Score (IPSS) $\geqslant 8$ were enrolled in this study. All participants completed questionnaires, including the IPSS and the Male Sexual Health Questionnaire (MSHQ), and serum prostate-specific antigen, transrectal ultrasound and uroflowmetry with post-void residual were measured. After initiating $0.2 \mathrm{mg}$ OD tamsulosin, patients were re-evaluated on the fourth and twelfth weeks of medication. The chi-squared test, the independent $t$-test and one-way ANOVA were used to compare means. Binary logistic regression analysis was used to calculate the odds ratio for all risk factors. A total of 177 men constituted the study cohort. No significant difference was observed between baseline and follow-up for the erectile function, ejaculatory function, satisfaction, sexual activity and desire domains (EFD, EjFD, SDA and ADD) or for erectile or ejaculatory bother mean scores. After 12 weeks, the overall incidence of ejaculatory dysfunction (EjD) was $13.4 \%$. Incidences of the seven different types of EjD (decreased frequency, delay, dryness, decreased strength/force, decreased volume, decreased pleasure and pain at ejaculation) were 2.4\%, 3.1\%, 3.9\%, 3.9\%, 6.3\%, 7.1\% and 3.1\%, respectively. Baseline EjFD scores were higher for IPSS responders than for non-responders (26.09 vs. 24.06, $P=0.03)$. An EjFD score reduction was more frequent in IPSS responders. The incidence of EjD was small, but not negligible and was more frequent in patients with less lower urinary tract symptoms, a smaller prostate, higher baseline MSHQ totals and higher EjFD scores.

Asian Journal of Andrology (2011) 13, 846-850; doi:10.1038/aja.2011.25; published online 25 July 2011

Keywords: alpha-1 adrenergic receptors; benign prostatic hyperplasia; ejaculation; lower urinary tract symptom; Male Sexual Health Questionnaire; prostatic hyperplasia; tamsulosin

\section{INTRODUCTION}

Benign prostatic hyperplasia (BPH) is highly prevalent in the aged male population. ${ }^{1,2} \alpha_{1}$-Adrenoceptor antagonists are commonly administered to treat $\mathrm{BPH}$ and their safety profiles are well-known to urologists. ${ }^{3-5}$ Tamsulosin is one such agent and has preferential selectivity and a greater affinity for $\alpha_{1 \mathrm{~A}^{-}}$adrenoceptors than for $\alpha_{1 \mathrm{~B}^{-}}$ adrenoceptors. ${ }^{5}$ Because $\alpha_{1 \mathrm{~A}}$-adrenoceptors are widely distributed in all organs that participate in the emission phase of ejaculation (epididymis, vas deferens, seminal vesicle, prostate gland, prostatic urethra and bladder neck), tamsulosin is suspected to play an inhibitory role during the ejaculatory emission phase. ${ }^{6}$ Tamsulosin also has a high affinity for $\mathrm{D}_{2}$-like and $5 \mathrm{HT}_{1 \mathrm{~A}}$ receptors, which play key roles in the central control of ejaculation, and thus is also suspected to have a central effect on ejaculation. ${ }^{6}$ Abnormal ejaculation is a typical side effect of tamsulosin. ${ }^{7,8}$ In a long-term, open-label, phase III extension study of $0.4 \mathrm{mg}$ tamsulosin once daily (OD), $30 \%$ of patients experienced ejaculatory dysfunction (EjD). ${ }^{5}$ Furthermore, the effects are dose-related, as demonstrated by a significantly higher incidence of $\mathrm{EjD}$ in men receiving $0.4 \mathrm{mg} \mathrm{OD}(8.4 \%)$ or $0.8 \mathrm{mg}$ OD $(18.1 \%)$ tamsulosin than in those receiving placebo $(0.2 \%)^{9}$

In South Korea, as in other Asian-Pacific countries, tamsulosin is prescribed at $0.2 \mathrm{mg} \mathrm{OD}$, and at this dosage, it may have an adverse effect on ejaculation. For example, in a Japanese study, tamsulosin at 0.2 or at $0.4 \mathrm{mg}$ OD for 3 days reduced mean ejaculatory volumes by $45 \%$ and $49 \%$, respectively, although no sperm were detected in midstream urine obtained after ejaculation. ${ }^{10}$ Nevertheless, the prevalence of EjD $(0.6 \%-4.5 \%)$ and of overall adverse events $(6.2 \%)$ after $0.2 \mathrm{mg}$ OD tamsulosin in South Korea is considerably lower than in Europe and the United States. ${ }^{11,12}$ However, in the studies mentioned above, EjD was not clearly defined or was sometimes reported as retrograde ejaculation. Furthermore, the degree of dysfunction has not been subject to study. Although a substantial number of papers reported prevalence values for various types of sexual dysfunction, further elaboration of the characteristics of EjD is lacking. ${ }^{13-15}$ Therefore, the aims of this study were to determine the degree of impact of $0.2 \mathrm{mg}$ OD

\footnotetext{
${ }^{1}$ Jeungpyung Health Center, Jeungpyung-gun, Chungbuk, 368-900, Korea; ${ }^{2}$ Department of Urology, Seoul Metropolitan Boramae Hospital, Seoul, 156-707, Korea; ${ }^{3}$ Aerospace Medical Center, Cheongwon, Chungbuk, 363-840, Korea; ${ }^{4}$ Catholic University of Korea College of Medicine, Seoul, 137-701, Korea; ${ }^{5}$ Korea University College of Medicine, Seoul, 152-703, Korea; ${ }^{6}$ Department of Urology, Yeungnam University College of Medicine, Daegu, 705-717, Korea; ${ }^{7}$ Department of Urology, Chonnam National University Medical School, Gwangju, 501-757, Korea; ${ }^{8}$ Department of Urology, Medical School and Institute for Medical Sciences, Chonbuk National University, Chonbuk, 561-712, Korea; ${ }^{9}$ Research Institute of Clinical Medicine and CTC for Medical Device of Chonbuk National University Hospital, Chonbuk, 561-712, Korea; ${ }^{10}$ Samsung Medical Center, Sungkyunkwan University School of Medicine, Seoul, 135-710, Korea; ${ }^{11}$ Gyeongsang National University, Gyeongnam, 660-702, Korea and ${ }^{12}$ Pusan National University College of Medicine, Pusan, 602-739, Korea

Correspondence: Dr H Son (volley@snu.ac.kr)
}

Received: 16 December 2010; Revised: 18 February 2011; Accepted: 14 March 2011; Published online: 25 July 2011 
tamsulosin for 12 weeks on sexuality, especially on ejaculatory function, and to evaluate voiding function efficacy after treatment with a validated, self-administered questionnaire on erection, ejaculation and sexual satisfaction, namely, the Male Sexual Health Questionnaire (MSHQ). In addition, we sought to identify risk factors for sexual dysfunction after tamsulosin treatment.

\section{MATERIALS AND METHODS}

\section{Participants}

Enrollment of the study subjects was conducted in urology clinics at nine university hospitals in South Korea from December 2008 to January 2010. Males who visited each urology clinic due to lower urinary tract symptoms (LUTS) suggestive of BPH and with an International Prostate Symptom Score (IPSS) of more than 8 were enrolled in this study. Patients were excluded if they had any of the following: a history of taking $\alpha$-blockers, $5 \alpha$-reductase inhibitors, antiandrogenic hormones or anticholinergics during the previous 4 weeks due to LUTS or BPH; received androgenic hormones or phosphodiesterase type 5 inhibitors during the previous 4 weeks due to erectile dysfunction; been treated using non-pharmacological methods for a prostate or urethral condition; a history of urinary tract infection, prostatitis, or a malignant neoplasm of the genitourinary tract; or severe hepatic or renal dysfunction.

\section{Study protocol}

Participants were evaluated at their first visit to the clinic via a detailed medical history, the recording of vital signs and a physical examination. In addition, they were asked to complete the IPSS, quality of life (QoL) survey and MSHQ; they were tested for serum prostate-specific antigen and underwent transrectal ultrasound and uroflowmetry with post-void residual volume.

Tamsulosin (0.2 mg OD) was administered orally for 12 weeks. Vital signs, physical examinations, IPSS, QoL, MSHQ and uroflowmetry with post-void residual were re-evaluated after 4 and 12 weeks of treatment (TW 4 and 12). Compliance with medication and possible adverse events were recorded at each visit. This study protocol was reviewed and approved by the Institutional Review Board.

\section{Definitions of sexual dysfunction and the effectiveness of tamsulosin medication}

The 25-item MSHQ is composed of a three-item erectile function domain (EFD), a seven-item ejaculatory function domain (EjFD), erection and ejaculation bother items, a six-item satisfaction domain (SAD), and a seven-item sexual activity and desire domain (ADD). ${ }^{16}$ Item scores (range: 0-5), total MSHQ scores and domain scores at baseline and at TW 4 and 12 were compared and differences were analysed. Erectile dysfunction was defined as an erectile domain score of $<6$ or a score of $<2$ for any item. EjD was classified into seven types of dysfunction using the seven-item ejaculatory function domain (decreased frequency, delay, dryness, decreased strength/force, decreased volume, decreased pleasure and pain at ejaculation). Dysfunction was defined as an item score of $<1$ at assessment or a decrease of $\geqslant 3$ points relative to the initial assessment. The number of subjects with each EjD divided by the overall number of subjects gave the incidence of each ejaculatory dysfunction.

Tamsulosin response in terms of LUTS suggestive of BPH was determined using IPSS/QoL and peak flow rate $\left(\mathrm{Q}_{\max }\right)$. Those with improvements of total IPSS and $\mathrm{Q}_{\max }$ of $\geqslant 25 \%$ or $\geqslant 30 \%$ from baseline were defined as IPSS and $\mathrm{Q}_{\max }$ responders, respectively. These definitions were suggested by previous studies. ${ }^{5,12,17}$ To evaluate associations between the occurrence of EjD and the degree of symptom improvement, we compared the MSHQ EjFD score variation differences after medication of tamsulosin responders and non-responders.

\section{Statistical analysis}

All statistical analyses were performed using SPSS version 13.0 (SPSS Inc., Chicago, IL, USA). The chi-squared test was used to compare categorical data, and the independent $t$-test and one-way ANOVA were used to compare numerical data. Wilcoxon's rank sum test and the Kruskal-Wallis test were used to compare nonparametric variables. Binary logistic regression analysis was used to calculate the odds ratios of all risk factors. All hypotheses were evaluated in a two-sided manner, and $P$ values of $<0.05$ were considered significant. Values are presented as means \pm s.d. unless otherwise indicated.

\section{RESULTS}

\section{Demographics}

Of the 213 subjects initially included in the study population, 29 were excluded because of incomplete data collection, and seven subjects were excluded because they were unable to ejaculate or lost ejaculate at their first visit to the clinic. As a result, 177 men composed the study cohort. General subject characteristics and baseline and follow-up data are included in Table 1. MSHQ total scores and domain scores are listed in Table 2.

\section{Primary end point}

For all 177 study subjects, no significant difference was observed between baseline and follow-up for erectile function, ejaculatory function, satisfaction, sexual activity and desire domains (EFD, EjFD, SDA, ADD) or for erectile or ejaculatory bother mean scores (Table 2 and Figure 1). We analysed relations between EjFD scores and item scores in detail. Figure 1 demonstrates that all EjFD item scores were the lowest (indicating more dysfunctional) at TW 12, but this was only significant for the pain item of the EjFD. The overall incidence of EjD at TW 12 determined using MSHQ EjFD scores was 13.4\%. The other incidences of ejaculatory dysfunction are listed in Table 3.

\section{Secondary end point}

IPSS responders comprised $39.0 \%$ and $49.2 \%$ of the 177 study subjects at TW 4 and 12. The mean increase of $\mathrm{Q}_{\max }$ from baseline was $2.00 \mathrm{ml} \mathrm{s}^{-1}$ at TW 12, and $\mathrm{Q}_{\max }$ responders comprised $18.6 \%$ of the study subjects. The results of efficacy parameters are listed in Table 1. MSHQ scores were not different between $Q_{\max }$ responders and non-responders. Mean baseline MSHQ total and EjD scores at TW 12 were greater for IPSS responders than for non-responders, but the difference was significant only for MSHQ EjD scores between responders and non-responders (83.83 vs. 80.27, $P=0.17$ and 26.09 vs. 24.06, $P=0.03$, respectively). A reduction in MSHQ domain score over 12 weeks was observed more frequently among IPSS responders than non-responders, but this difference was significant for EjFD scores only ( $42.5 \%$ vs. $25.5 \%, P=0.017$ ) (Figure 2 ).

\section{Risk factors for EjD}

MSHQ EjD scores were reduced in 60 subjects, and these patients showed higher mean baseline MSHQ total and EjFD scores than subjects whose scores were not reduced, but the differences were not statistically significant $(83.14$ vs. $81.53, P=0.56 ; 25.73$ vs. 24.71 , $P=0.32$ ). Furthermore, the mean prostate volume of these subjects 
Table 1 Demographics at baseline and follow-up $(n=177)$

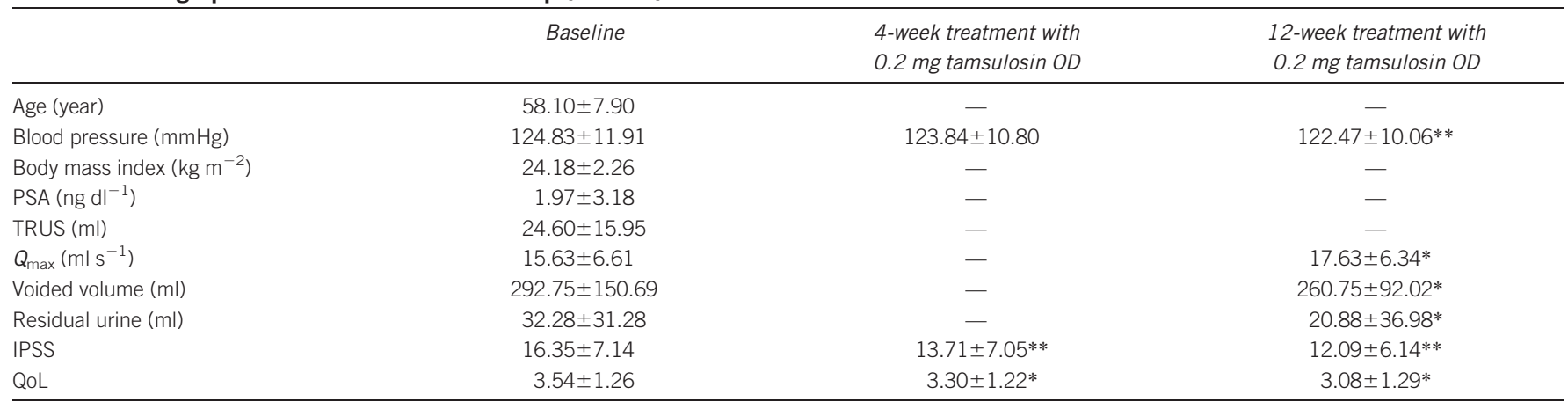

Abbreviations: IPSS, International Prostatic Symptom Score; OD, once daily; QoL, quality of life; PSA, prostate-specific antigen; $Q_{\text {max }}$, peak urinary flow rate; TRUS, transrectal ultrasonography.

Values are presented as mean \pm s.d.

$* P<0.05$, compared with the baseline values.

$* * P<0.01$, compared with the baseline values.

Table 2 MSHQ total and domain scores at baseline and follow-up $(n=177)$

\begin{tabular}{lccc}
\hline & Baseline & 4 weeks & 12 weeks \\
\hline MSHQ total & $82.06 \pm 17.05$ & $82.12 \pm 18.19$ & $81.07 \pm 19.11$ \\
Erection domain & $9.74 \pm 3.33$ & $9.67 \pm 3.35$ & $9.60 \pm 3.43$ \\
Ejaculation domain & $25.06 \pm 6.41$ & $24.60 \pm 6.81$ & $23.76 \pm 7.87$ \\
Satisfaction domain & $19.49 \pm 4.52$ & $19.53 \pm 4.83$ & $19.28 \pm 4.64$ \\
Activity and desire & $12.05 \pm 2.62$ & $12.36 \pm 2.65$ & $12.28 \pm 2.57$ \\
$\quad$ domain & & & \\
\hline
\end{tabular}

Abbreviations: MSHQ, Male Sexual Health Questionnaire.

Values are presented as mean \pm s.d. Mean score differences are not significant in paired-sample $t$-tests.

was smaller ( $21.58 \mathrm{ml}$ vs. $26.13 \mathrm{ml}, P=0.085)$, the voided volume was larger (329.95 ml vs. $268.51 \mathrm{ml}, P=0.037), \mathrm{Q}_{\max }$ was faster $\left(17.67 \mathrm{ml} \mathrm{s}^{-1}\right.$ vs. $14.59 \mathrm{ml} \mathrm{s}^{-1}, P=0.003$ ), and PVR was smaller (26.45 vs. 35.27 , $P=0.076)$ compared to subjects with a non-reduced MSHQ EjD score. EjFD was found to be the only significant risk factor for EjD occurrence by binary logistic regression analysis $(P<0.001)$. The odds ratio for EjD

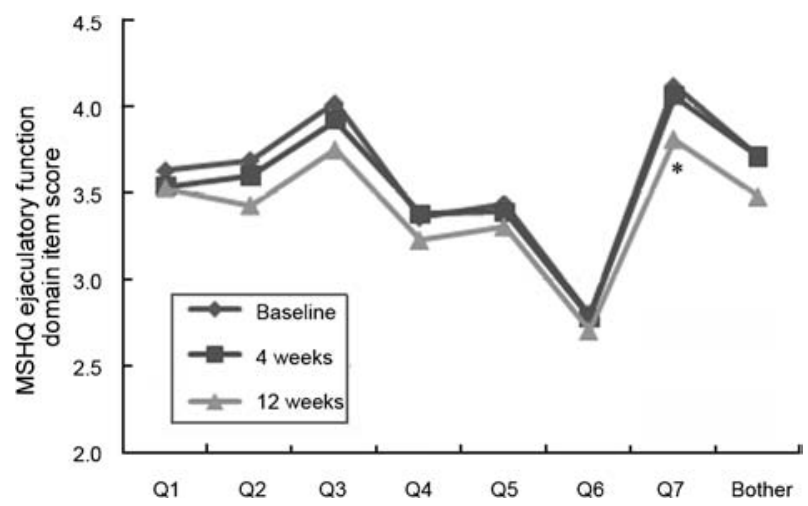

Figure 1 Plots demonstrating MSHQ ejaculatory function domain item scores evaluated at baseline and after 4 and 12 weeks of treatment. Differences were not statistically significant except for the seventh item (pain) at 12 weeks of treatment $(P=0.013)$. Q1-Q7: ejaculatory function domain item numbers 1-7; Bother: MSHQ ejaculatory bother item score. MSHQ, Male Sexual Health Questionnaire.
Table 3 Incidence of ejaculatory dysfunction (\%)

\begin{tabular}{lcc}
\hline Ejaculatory dysfunction & 4 weeks & 12 weeks \\
\hline Decreased frequency & 3.1 & 2.4 \\
Delay of ejaculation & 3.9 & 3.1 \\
Dryness (anejaculation) & 2.4 & 3.9 \\
Decreased strength of ejaculation & 4.7 & 3.9 \\
Decreased volume of ejaculation & 5.5 & 6.3 \\
Decreased pleasure & 3.1 & 7.1 \\
Pain on ejaculation & 2.4 & 3.1 \\
Total & 14.2 & 13.4 \\
\hline
\end{tabular}

was 10.14 (95\% CI: 2.245-45.857) in patients with a baseline EjFD score of $\leqslant 26$.

\section{DISCUSSION}

This is the first multicentre study to evaluate the impact of tamsulosin at $0.2 \mathrm{mg}$ OD for 12 weeks on ejaculatory function using a validated questionnaire (the MSHQ). By using this questionnaire, we were able to evaluate $\mathrm{EjD}$ with respect to frequency, volume and other variables. Furthermore, the occurrence of these dysfunctions was found to be associated with the degree of LUTS improvement.

In a previous study conducted over a 53-week treatment period, EjD or abnormal ejaculation was reported in $10 \%$ and $26 \%$ of patients on 0.4 or $0.8 \mathrm{mg}$ of tamsulosin OD, respectively. ${ }^{18}$ In another study, over a mean treatment duration of 64.5 weeks, $30 \%$ of patients treated with tamsulosin (0.4-0.8 mg OD) reported abnormal ejaculation. ${ }^{5}$ However, in these studies, EjD and abnormal ejaculation were assessed using patient self-reports, and the definitions used were less than clear. It has sometimes been assumed that abnormal ejaculation associated with $\alpha$-blocker use represents retrograde ejaculation in an analogous manner to the retrograde ejaculation encountered after transurethral resection of the prostate. ${ }^{8,18}$ However, in two recent studies, researchers measured ejaculate and post-ejaculation urine volumes and demonstrated that $\alpha$-blocker-associated abnormal ejaculation may represent anejaculation rather than retrograde ejaculation. ${ }^{10,19}$ Hellstrom and Sikka ${ }^{19}$ reported that ejaculatory function in subjects on tamsulosin $0.8 \mathrm{mg}$ OD was marked by a reduced ejaculate volume in almost $90 \%$ of subjects and that anejaculation was present in approximately $35 \%$ of participants. ${ }^{19}$ In the present study, we found that anejaculation, decreased ejaculate volume 
a

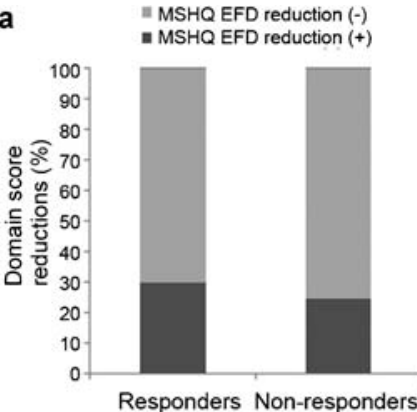

C

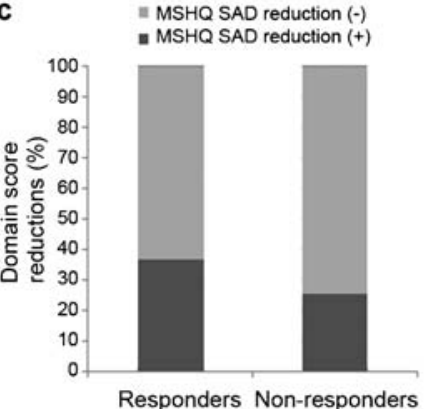

b

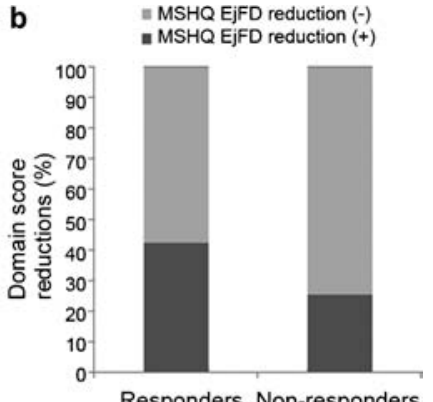

d

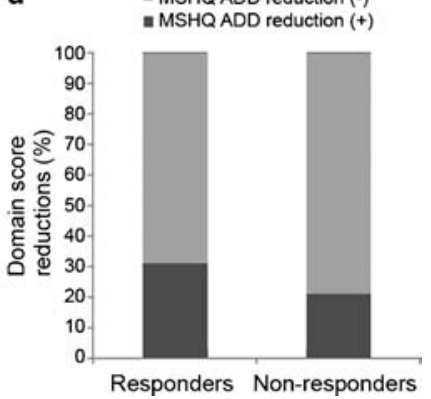

Figure $2 \mathrm{MSHQ}$ domain score reductions after 12 weeks of tamsulosin treatment were more common among IPSS responders than IPSS non-responders, but this difference was significant for EjFD scores only $(42.5 \%$ vs. $25.5 \%$, $P=0.017$ ). (a) Erectile function domain (EFD); (b) ejaculatory function domain (EjFD); (c) satisfaction domain (SAD); (d) activity and desire domain (ADD). IPSS, International Prostatic Symptom Score; MSHQ, Male Sexual Health Questionnaire.

and overall EjD occurred in 3.9\%, 6.3\% and $13.4 \%$ of participants, respectively, on tamsulosin $0.2 \mathrm{mg}$ OD at 12 weeks follow-up (Table 3). This incidence of EjD on tamsulosin $0.2 \mathrm{mg}$ is considered to be in direct proportion to that on $0.8 \mathrm{mg}$. Therefore, the incidence of EjD may well be dose-related rather than being due to a genetic or racial difference in the affinity or in the selectivity of tamsulosin for $\alpha_{1}$-adrenoceptors. However, researchers in Japan showed that the frequency distribution of the $\alpha_{1}$-adrenoceptor polymorphism is significantly different in Japanese than in Americans. ${ }^{20}$ Thus, the possibility of an association between tamsulosin-induced EjD and the $\alpha_{1}$-adrenoceptor gene polymorphism in Koreans remains to be investigated.

A combined analysis of 12 European and US clinical studies found that BPH symptom improvements were slightly greater in patients with an ejaculation problem than in those without. ${ }^{21}$ In addition, these patients were found to be less likely to discontinue treatment than patients without an ejaculation problem. Our finding that MSHQ EjFD scores were reduced more frequently among IPSS responders than non-responders is consistent with this previous finding. This could be explained by a greater selectivity and/or affinity of tamsulosin for the $\alpha_{1 \mathrm{~A}}$-adrenoceptors and the distribution of these receptors, which are well expressed in the organs that participate in the ejaculatory emission phase. ${ }^{5}$

In addition, we found that tamsulosin-induced EjD was more frequent among patients with a relatively small prostate volume, a faster $\mathrm{Q}_{\max }$, a smaller residual urine volume, and higher baseline MSHQ total and EjFD scores. Although age differences between the subjects with and without EjFD score reductions were not significant (57.97 years $v s .58 .34$ years) in our study, this effect had been reported in other studies. A pooled analysis of European phase III studies found abnormal ejaculation to be more prevalent among patients aged $<65$ years $(6.3 \%)$ than in those aged $\geqslant 65$ years $(2.6 \%)$, but that incidences among those treated with a placebo were similar in these age groups $(1.0 \%-$ $1.1 \%) .^{22}$ Furthermore, the highest incidence of abnormal ejaculation ever reported was in a clinical pharmacology study of 25-year-old volunteers. ${ }^{19}$ Meanwhile, in the present study, IPSS and MSHQ EjFD scores were found to be significantly correlated (correlation coefficient $=-0.226, P=0.004$ at TW 4; correlation coefficient $=-0.207$, $P=0.011$ at TW 12). Based on our findings, we believe that patients with mild LUTS and good ejaculatory function are more likely to suffer from abnormal ejaculation after tamsulosin treatment.

This study is limited because it was not case-controlled or comparative and because we did not investigate dose responsiveness at higher tamsulosin doses. In addition, we did not measure ejaculate volumes, but rather relied on patient self-assessments in the MSHQ. However, measuring ejaculate volumes in the clinical situation is inconvenient. Instead, we suggest that clinicians use the MSHQ to obtain information about EjD.

Using the MSHQ, we were able to investigate $\mathrm{EjD}$ with respect to frequency, volume, pleasure and other variables after administering tamsulosin at $0.2 \mathrm{mg}$ daily for 12 weeks. The incidence of EjD was small but not negligible. We found that the occurrence of EjD was associated with the degree of LUTS improvement. Furthermore, patients with a relatively small prostate volume, a greater $\mathrm{Q}_{\max }$ a smaller PVR, and higher baseline MSHQ total and EjFD scores were found to suffer more frequently from abnormal ejaculation after receiving tamsulosin at $0.2 \mathrm{mg}$ daily. However, abnormal ejaculation is found in more than $50 \%$ of men aged over 60 years, and thus, the clinical significance of the small increase in prevalence in older men requires further investigation.

\section{AUTHOR CONTRIBUTIONS}

SSH, PJK and SH conceived of the study, participated in the design of the study, performed the statistical analysis and drafted the manuscript. KKT, KSW, MDG, MKH, PK, LSW, HJS and PNC participated in the design and coordination of the study, performed participant recruitment and helped to draft the manuscript. All authors read and approved the final manuscript.

\section{COMPETING FINANCIAL INTERESTS}

The authors declare no competing financial interests.

1 Lee E, Yoo KY, Kim Y, Shin Y, Lee C. Prevalence of lower urinary tract symptoms in Korean men in a community-based study. Eur Urol 1998; 33: 17-21.

2 Garraway WM, Collins GN, Lee RJ. High prevalence of benign prostatic hypertrophy in the community. Lancet 1991; 338: 469-71.

3 Lepor $\mathrm{H}$. Phase III multicenter placebo-controlled study of tamsulosin in benign prostatic hyperplasia. Tamsulosin Investigator Group. Urology 1998; 51: 892900.

4 Narayan P, Tewari A. A second phase III multicenter placebo controlled study of 2 dosages of modified release tamsulosin in patients with symptoms of benign prostatic hyperplasia. United States 93-01 Study Group. J Urol 1998; 160: 1701-6.

5 Narayan P, Lepor H. Long-term, open-label, phase III multicenter study of tamsulosin in benign prostatic hyperplasia. Urology 2001; 57: 466-70.

6 Giuliano F. Impact of medical treatments for benign prostatic hyperplasia on sexual function. BJU Int 2006; 97 (Suppl 2) 34-8.

7 Rosen RC, Giuliano F, Carson CC. Sexual dysfunction and lower urinary tract symptoms (LUTS) associated with benign prostatic hyperplasia (BPH). Eur Urol 2005; 47: 824-37.

8 van Dijk MM, de la Rosette JJ, Michel MC. Effects of alpha ${ }_{1}$-adrenoceptor antagonists on male sexual function. Drugs 2006; 66: 287-301. 
9 Flomax (tamsulosin hydrochloride) [prescribing information]. Ridgefield, CT: Boehringer Ingelheim Pharmaceuticals, Inc.; 2002

10 Hisasue S, Furuya R, Itoh N, Kobayashi K, Furuya S et al. Ejaculatory disorder caused by alpha- 1 adrenoceptor antagonists is not retrograde ejaculation but a loss of seminal emission. Int J Urol 2006; 13: 1311-6.

11 Park CH, Chang HS, Oh BR, Kim HJ, Sul CK et al. Efficacy of low-dose tamsulosin on lower urinary tract symptoms suggestive of benign prostatic hyperplasia: a nonblind multicentre Korean study. Clin Drug Invest 2004; 24: 41-7.

12 Chung BH, Roehrborn CG, Siami P, Major-Walker K, Morrill BB et al. Efficacy and safety of dutasteride, tamsulosin and their combination in a subpopulation of the CombAT study: 2-year results in Asian men with moderate-to-severe BPH. Prostate Cancer Prostatic Dis 2009; 12: 152-9.

13 Lewis RW. Epidemiology of sexual dysfunction in Asia compared to the rest of the world. Asian J Androl 2011; 13: 152-8.

14 Park HJ, Park JK, Park K, Lee SW, Kim SW et al. Prevalence of premature ejaculation in young and middle-aged men in Korea: a multicenter internet-based survey from the Korean Andrological Society. Asian J Androl 2010; 12: 880-9.

15 Son H, Song SH, Kim SW, Paick JS. Self-reported premature ejaculation prevalence and characteristics in Korean young males: community-based data from an internet survey. J Androl 2010; 31: 540-6.
16 Rosen RC, Catania J, Pollack L, Althof S, O'Leary M et al. Male Sexual Health Questionnaire (MSHQ): scale development and psychometric validation. Urology 2004; 64: 777-82.

17 Narayan P, Bruskewitz R. A comparison of two phase III multicenter, placebocontrolled studies of tamsulosin in BPH. Adv Ther 2000; 17: 287-300.

18 Debruyne FM. Alpha blockers: are all created equal? Urology 2000; 56: 20-2.

19 Hellstrom WJ, Sikka SC. Effects of acute treatment with tamsulosin versus alfuzosin on ejaculatory function in normal volunteers. J Urol 2006; 176: 1529 33.

20 Shibata K, Hirasawa A, Moriyama N, Kawabe K, Ogawa S et al. Alpha 1a-adrenoceptor polymorphism: pharmacological characterization and association with benign prostatic hypertrophy. Br J Pharmacol 1996; 118: 1403-8.

21 Speakman MJ SR, Anthonijs G, Doyle CA. Patients on tamsulosin experiencing abnormal ejaculation choose to remain longer in clinical trials and have slightly better improvement in symptom score than other patients [abstract]. J Urol 2003; 169: 334.

22 Chapple CR, Baert L, Thind P, Hofner K, Khoe GS et al. Tamsulosin 0.4 mg once daily: tolerability in older and younger patients with lower urinary tract symptoms suggestive of benign prostatic obstruction (symptomatic BPH). The European Tamsulosin Study Group. Eur Urol 1997; 32: 462-70. 